\title{
Hearing Loss Gene Panel Testing: Considerations for Clinical Practice
}

\author{
Alpana M Kulkarni* \\ Paediatric Audiology, Hertfordshire Community NHS Trust, UK
}

*Corresponding author: Alpana Kulkarni, Consultant in Audiovestibular Medicine, Peace Children's centre, Peace Prospect, Watford WD17 3EW, United Kingdom.

Received Date: September 12, 2020

Published Date: September 24, 2020

\begin{abstract}
Hearing loss gene panel testing has now been introduced into mainstream NHS (National Health Service) as genetic test which can be offered to families with child with hearing loss. Clinicians requesting this test need to have an understanding of its sensitivity, specificity of this test and its limitations and challenges. They also need to be aware of the ethical issues which may arise by undertaken this test and counsel families accordingly before requesting this test.
\end{abstract}

Keywords: Sensorineural hearing loss; Gene panel testing

\section{Introduction}

1 in 1000 children is born with sensorineural hearing loss. 2.7 in 1000 children develop hearing loss in late childhood and the incidence increases to 3.5 in 1000 in adolescence [1]. Genetic diagnosis of hearing loss is important from prognostic point of view, offering advice for suitable intervention such as cochlear implant in Connexin 26 deafness, or oral speech with cochlear implant in Usher's syndrome due to retinitis pigmentosa. Around $50 \%$ of hearing loss is genetic in origin and of these at least $20 \%$ is syndromic and $80 \%$ is non-syndromic [2]. Syndromic loss can be identified with single gene testing using Sanger sequencing approach, for example EYA1 mutation in Branchio-Oto-Renal syndrome. This condition clinically manifests through phenotypical changes such as preauricular pits or tags along with sensorineural or mixed hearing loss. Similarly, testing for Connexin 26, which is the commonest cause of non-syndromic hearing loss (NHSL) and responsible for $\leq 50 \%$ of non-syndromic autosomal recessive loss, is currently requested by single gene testing in the UK [3]. However, if single gene testing is negative then no conclusion is reached about the aetiology of the deafness. In some cases, sequential single gene tests may be carried out for different genes but this is expensive.

Genomics England was initially set up to deliver the '100,000 Genomic Project' and further funding has been given to expand the UK's NHS biobank to sequence one million whole genomes in the next five years. Thus, the genetic laboratory services in the UK have rapidly expanded to meet this demand. With next generation sequencing, also called 'massive parallel sequencing', it would be possible to test for millions of DNA fragments in one reaction. Thus, technology which target and enrich specific areas of the genome coupled with massive parallel sequencing is now the new norm for genetic testing for hearing loss. The genetic labs are now offering non-syndromic and syndromic hearing panel testing using this technology. With the hearing panel testing, currently more than 100 genes (both non syndromic and syndromic) can be tested for any pathologic variants and with turnover time of 12 to 14 weeks, a genetic diagnosis can benefit a child and the family with hearing loss. 


\section{What is in the literature?}

Shearer, et al. [2010] [4] tested 9 patients with hearing loss to evaluate the feasibility of massive parallel sequencing by testing the exons of all genes involved in NSHL. 605 single nucleotide polymorphisms (SNP) were genotyped by Sanger sequence to test the sensitivity and specificity of the parallel sequencing technologies. Causative mutations were identified in all positive but not negative controls indicating that this technology can be used successfully to identify mutations in hearing loss [4]. Subsequently many studies have demonstrated the usefulness of this methodology.

Shearer, et al. [2016] carried out a literature review to evaluate the use of new genetic techniques for genetic diagnosis of hearing loss. They studied 20 studies which included 426 control samples and 603 patients with unknown hearing loss. Control analysis showed $>99 \%$ sensitivity and specificity for clinical use of massive parallel sequencing tests. They identified that the overall diagnostic rate was $41 \%$ (range of $10 \%$ to $83 \%$ ) and it varied due to factors, such as inheritance and pre-screening prior to testing [5]. A further study by Cabanillas, et al. 2018 [6] developed a panel with 199 genes including syndromic and non-syndromic sensorineural hearing loss and demonstrated a diagnostic yield of $42 \%$ in patients with sensorineural hearing loss [6].

It is important however to develop the right panel of genes and precise targeted genomic areas to improve the sensitivity, the challenges are to interpret the variants with appropriate clinical data. So, it is vital that there is a close collaboration between the Clinician who is requesting the test with the genetic lab scientist. A comprehensive medical and family history should be provided with the request. Study by Christina M Sloan Heggen, et al. [2016] [7] performed comprehensive clinical genetic testing with massive parallel sequencing on 1119 sequential patients. Yield of the genetic cause of hearing loss was found in 39\% (440) patients. The diagnostic yield was highest considerably based on phenotype and was highest for patients with positive family history [7].

\section{Personal practice}

In my clinical practice I have instigated this panel testing as second line in families where more than one child has been identified with non-syndromic autosomal recessive hearing loss. So far three families each with two affected siblings have been tested and they all have been identified with pathogenic mutations. It will be useful to have an algorithm depending on the characteristics of the Clinicians case load based on ethnic groups, family history and phenotypical presentations. For example, if an MRI of the inner ears shows widened vestibular aqueduct then single gene testing with Pendrin gene is more appropriate, whereas if two children are affected in the same family with normal parents and normal MRI, then hearing panel testing should be instigated (see attached Algorithm as an example).

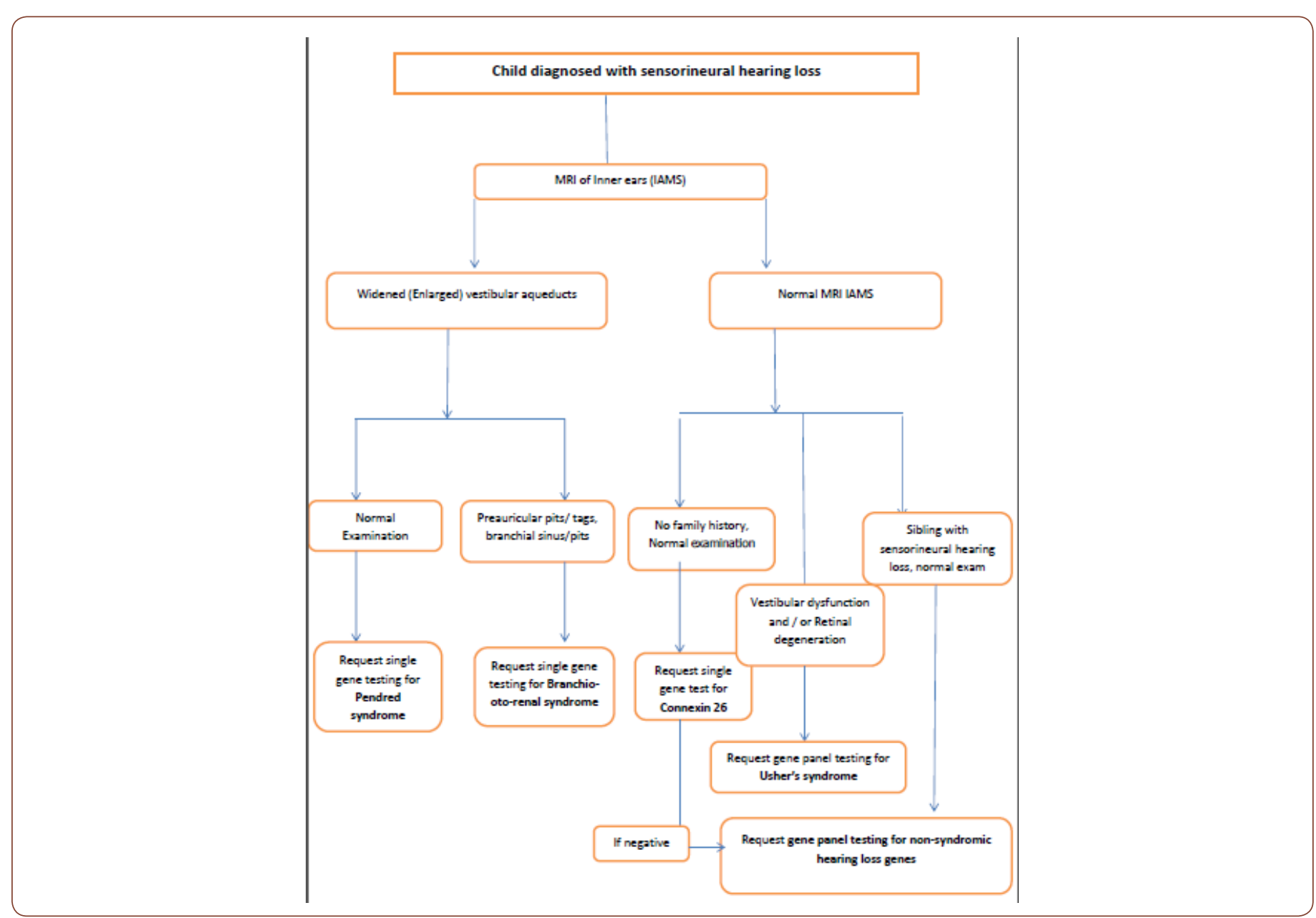




\section{Ethical considerations}

Clinicians need to be aware of the merits as well as demerits of this gene panel test and counsel parents accordingly. Some results may be unexpected, variants in genes unrelated to hearing loss may be uncovered and in some cases results may be very upsetting to patients/parents.

\section{Conclusion}

Hearing loss gene panel is now well established sensitive test which can improve the diagnostic yield and provide a genetic diagnosis to a child/patient with sensorineural hearing loss. However, to improve the sensitivity and reduce patient anxiety and unexpected or unnecessary results it is vital that requesting clinician should have a good knowledge of the genetic and other aetiological investigations of sensorineural hearing loss and should provide as much phenotype information to the genetic scientist as possible to assist interpretation and establish causality of these results. Having an algorithm for which patients to test, will also help improve sensitivity of the testing and be more cost effective to the Clinician.

\section{Acknowledgement}

None.

\section{Conflict of Interest}

None.

\section{References}

1. Morton CC, Nance WE (2006) Newborn hearing screening--a silent revolution. N Engl J Med 354(20): 2151-2164.

2. Korver AM, Smith RJ, Van Camp G, Mark R Schleiss, Maria AK Bitner Glindzicz, et al. (2017) Congenital hearing loss. Nat Rev Dis Primers 3: 16094.

3. Snoeckx RL, Huygen PL, Feldmann D, Sandrine Marlin, Françoise Denoyelle, et al. (2005) GJB2 mutations and degree of hearing loss: a multicenter study. Am J Hum Genet 77(6): 945-957.

4. Shearer AE, DeLuca AP, Hildebrand MS, Kyle R Taylor, José Gurrola, et al. (2010) Comprehensive genetic testing for hereditary hearing loss using massively parallel sequencing. Proc Natl Acad Sci U S A 107(49): 2110421109.

5. Shearer AE, Smith RJ (2015) Massively Parallel Sequencing for Genetic Diagnosis of Hearing Loss: The New Standard of Care. Otolaryngol Head Neck Surg 153(2): 175-182.

6. Cabanillas R, Diñeiro M, Cifuentes GA, David Castillo, Patricia C Pruneda, et al. (2018) Comprehensive genomic diagnosis of non-syndromic and syndromic hereditary hearing loss in Spanish patients. BMC Med Genomics 11(1): 58.

7. Sloan Heggen CM, Bierer AO, Shearer AE, Diana L Kolbe, Carla J Nishimura, et al. (2016) Comprehensive genetic testing in the clinical evaluation of 1119 patients with hearing loss. Hum Genet 135(4): 441-450. 